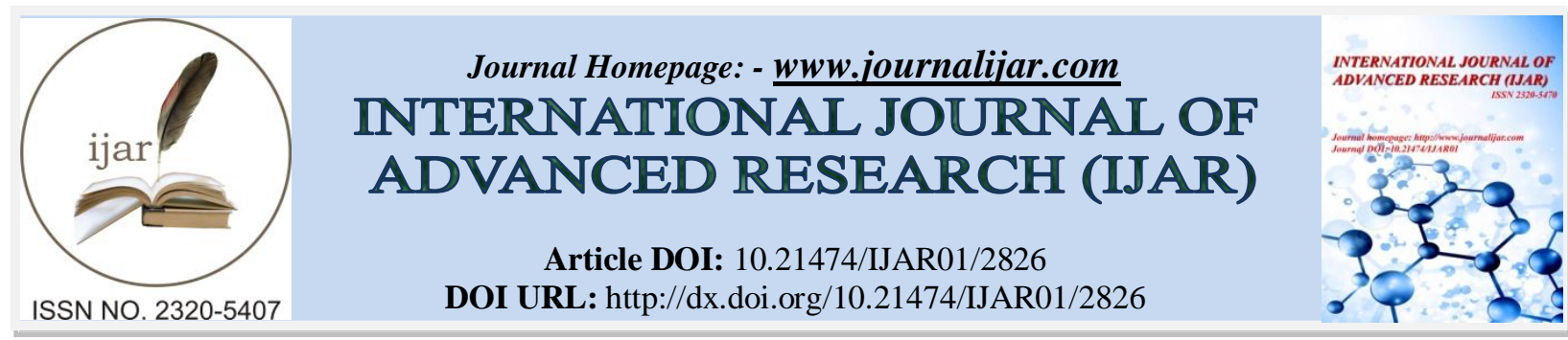

RESEARCH ARTICLE

\title{
TRAITEMENT CHIRURGICAL DE LA RUPTURE DU LIGAMENT CROISE ANTERIEUR CHEZ L'ENFANT SELON LA TECHNIQUE DE MAC-INTOSH MODIFIEE JAEGER (A PROPOS D'UNE SERIE CONTINUE DE 23 CAS).
}

Jalal Boukhris, Mojib Rifi, Sami Mezghani, Jean Henri Jaeger.

Service de Chirurgie Orthopédique du Genou et Traumatologie du Sport- CHU Strasbourg.

\section{Manuscript Info} anc.................

\section{Manuscript History}

Received: 17 November 2016

Final Accepted: 18 December 2016

Published: January 2017

\section{Key words:-}

Anterior cruciate ligament - Mac Intosh

- Ligamentoplasty - Fascia lata -

Cartilage growth

\section{Abstract}

Waiting for the end of growth for treating lesions of the anterior cruciate ligament seems to be mistake. Inadequate results of conservative treatment can harm the harmonious development of the child, thus surgical treatment has become a necessity. A conventional ligament as embodied in adults is not possible in children because of the risks of secondary growth disorder. With our experience in adults of mixed tuck the fascia lata using the technique of modified MacIntosh amended Jaeger, we have adapted this technique to the child respecting the growth of cartilage. We report the experience of the surgical management of 23 children in our institution between January 2000 and December 2009, according to the technique of Jaeger and their followed and we discuss our results by comparing to literature data recent.

Copy Right, IJAR, 2016,. All rights reserved.

\section{Introduction:-}

La fréquence et la gravité des lésions du ligament croisé antérieur de l'enfant ont longtemps été minimisées. Devant l'évolution défavorable de nombreuses lésions traitées de manière conservatrices, le traitement chirurgical est devenu une nécessité. Une ligamentoplastie conventionnelle telle qu'elle est réalisée chez l'adulte n'est pas envisageable chez l'enfant du fait des risques de troubles de croissance secondaires. Fort de notre expérience chez l'adulte de la plastie mixte au fascia-lata selon la technique de Mac-Intosh modifiée Jaeger, nous avons adapté cette technique à l'enfant en respectant le cartilage de croissance.

\section{Methodes:-}

\section{Description de la technique [1]:-}

L'intervention est précédée d'un examen clinique systématique réalisé sous anesthésie général. Il confirme le diagnostic et précise avec exactitude l'importance des laxités. Il facilite la mise en évidence d'un ressaut rotatoire. Ces recherches peuvent être difficiles sans anesthésie, particulièrement chez l'enfant.

Le jeune patient est ensuite installé en décubitus dorsal, le genou maintenu à $80^{\circ}$ de flexion par un appui placé à la racine de la cuisse sous le garrot pneumatique. L'intervention débute par la réalisation d'une mini-arthrotomie antéro-médiale de 3 à $4 \mathrm{~cm}$ (Fig.1). L'examen de la cavité articulaire permet un bilan lésionnel précis, les ménisques sont testés à l'aide d'un crochet, un geste chirurgical approprié sera réalisé devant une lésion méniscale,

Corresponding Author:- Jalal Boukhris.

Address:- Service de Chirurgie Orthopédique du Genou et Traumatologie du Sport- CHU Strasbourg. 
malheureusement fréquente chez l'enfant, en évitant par tous les moyens une méniscectomie même partielle. Enfin les surfaces du cartilage seront vérifiées.

L'incision est prolongée distalement afin de repérer le cartilage de croissance à l'aide d'aiguilles fines. Le tunnel tibial doit respecter ce cartilage. Son point d'entrée sera proximal par rapport au cartilage fertil tibial et situé au bord médial de la tubérosité antérieure tibiale. Son trajet aboutit au pied du LCA rompu, son diamètre de 4,5 à $6 \mathrm{~mm}$ sera adapté à l'âge de l'enfant (Fig.2).

L'incision cutanée latérale débute en regard du tubercule infra-condylien tibial et se prolonge sur la face latérale de la cuisse (Fig.3). Le transplant du fascia-lata est prélevé en respectant son insertion distale au tubercule infracondylien tibial, sa largeur est de $10 \mathrm{~mm}$ en distal et de 30 à $40 \mathrm{~mm}$ en proximal. La longueur du transplant varie de 10 à $15 \mathrm{~cm}$ en fonction de la morphologie de l'enfant. La bandelette du fascia-lata est tubulisée sur ses derniers centimètres (Fig.4).

Pour faciliter la fermeture sans tension du fascia-lata, le muscle vaste latéral est decollé du septum intermusculaire latéral, jusqu'à son insertion fémoral, le septum est alors sectionné au raz du fémur. Le ligament collatéral fibulaire est repéré et disséqué. Une rigole condylienne fémorale est réalisé en sous périosté, son point d'entrée se situe en arrière de l'insertion fémorale du ligament collatéral fibulaire, son trajet est réalisé au ciseau de smilie jusqu'à la face médiale du condyle latéral, cette rigole respecte le cartilage de croissance fémoral distal en étant plus proximal (Fig.5).

Par l'arthrotomie antéro-médiale, on perfore la capsule articulaire postérieure afin de préparer le passage du transplant (Fig.6).Il est ensuite récupéré dans l'articulation à travers la brèche capsulaire postérieure. Le transplant est enfin passé dans le tunnel tibial. Sa tension est réglé par traction manuelle le pied en rotation neutre, genou fléchi à $80^{\circ}$. Sa fixation est assurée par une agrafe métallique crantée, positionnée sous le cartilage de croissance métaphysaire tibial (Fig.7).

Le trajet du transplant comporte donc une partie extra-articulaire et une partie intra-articulaire assurant une stabilisation antérieure optimale tout en respectant le cartilage de croissance. Avant la fermeture, on contrôle l'absence de déficit de mobilité en flexion et en extension. L'absence de tiroir antérieur en flexion et en subextension est contrôlée. La fermeture du fascia-lata est réalisée afin de réparer le hauban extérieur fondamental pour la stabilité du genou. Les incisions cutanées sont suturées par surjet intra-dermiques. L'appui complet est autorisé. Le protocole de rééducation sera adapté à l'âge de l'enfant.

\section{Description de la série:-}

Il s'agit d'une étude rétrospective portant sur 23 enfants opérés au sein de notre établissement selon la technique de Jaeger entre Janvier 2000 et Décembre 2009. Notre série se composait de 18 garçons et 5 filles, souffrant d'instabilité post-traumatique du genou retentissant sur leurs activités physiques. 15 patients pratiquaient un sport de type pivot ou pivot-contact, dont 13 en compétition. Les scores préopératoires de Lysholm et de Tegner étaient respectivement de $74(58-90)$ et 8,1 (4 à 9). Nous avons opéré 17 genoux droits et 5 genoux gauches. Les patients ont été opérés par deux chirurgiens du service selon la même technique, décrite précédemment. Les détails techniques opératoires ont été soigneusement notés, ainsi que les traitements complémentaires et la chronologie de la reprise des activités sportives.

\section{Resultats:-}

La durée moyenne du garrot a été de 42,5 min (35 - 50). L'exploration articulaire a permis d'objectiver des lésions chondrales chez 1 patient et de traiter 4 lésions méniscales en zone avasculaire (blanche-blanche). La sortie du service s'est effectuée en moyenne au $7^{\text {ème }}$ jour post-opératoire $(8-9)$ en centre de rééducation pour la majorité des patients ou à domicile directement.

Au premier jour post-opératoire les douleurs sur une échelle visuelle analogique sont en moyenne à $4(1-6)$. Deux patients se plaignaient de gonflement du genou opéré à l'effort. . Aucun patient n'a rapporté d'épisode d'instabilité. La flexion est supérieure à $130^{\circ}$ pour tous les patients à 6 mois post opératoire. A 6 semaines un déficit d'extension chiffré à $1,15^{\circ}$ est retrouvé chez 1 patient, sans qu'il y ait de difference avec le programme de rééducation entre les patients. Aucun patient n'a de ressaut rotatoire. Un arrêt dur, retardé, au test de Trillat-Lachman à été retrouvé chez 5 patients $(21,7 \%)$ à un an. Tous les patients ont bénéficié d'un test isocinétique à trois et six mois. Le score IKDC 
fonctionnel subjectif moyen était de 80,3\% (41 à 100\%). Le Lysholm Knee Score moyen était de 83\% (31 à 100\%). Tous les patients ont eu une radiographie du genou opéré au $6^{\text {ème }}$ mois post-opératoire.

Nous avons recensé un hématome de cuisse qui s'est résorbé spontanément au huitième jour post opératoire, un sepsis superficiel traité par 15 jours d'antibiothérapie per os (Pristinamycine) et soins locaux, La mesure des laxités au KT 2000, chez les 42 patients revus en consultation dont le genou controlatéral était sain, a retrouvé une laxité différentielle moyenne de $2,1 \mathrm{~mm}(0$ à $6 \mathrm{~mm})$.

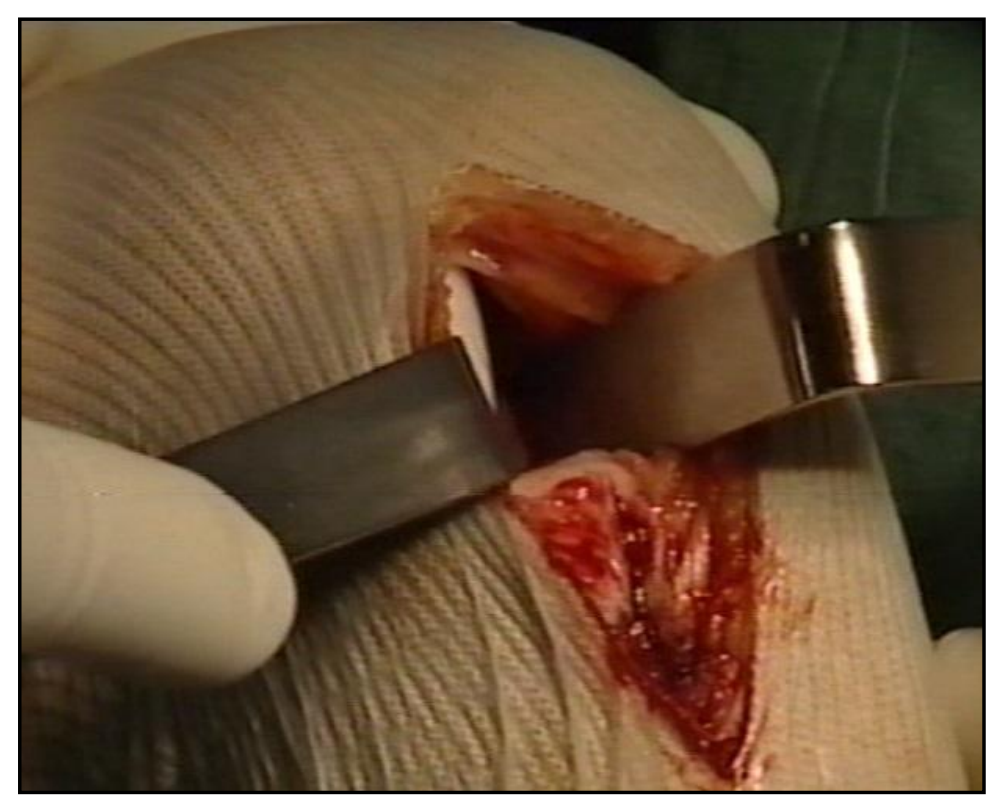

Fig.1:- Mini-arthrotomie antéro-médiale et exploration de la cavité articulaire.

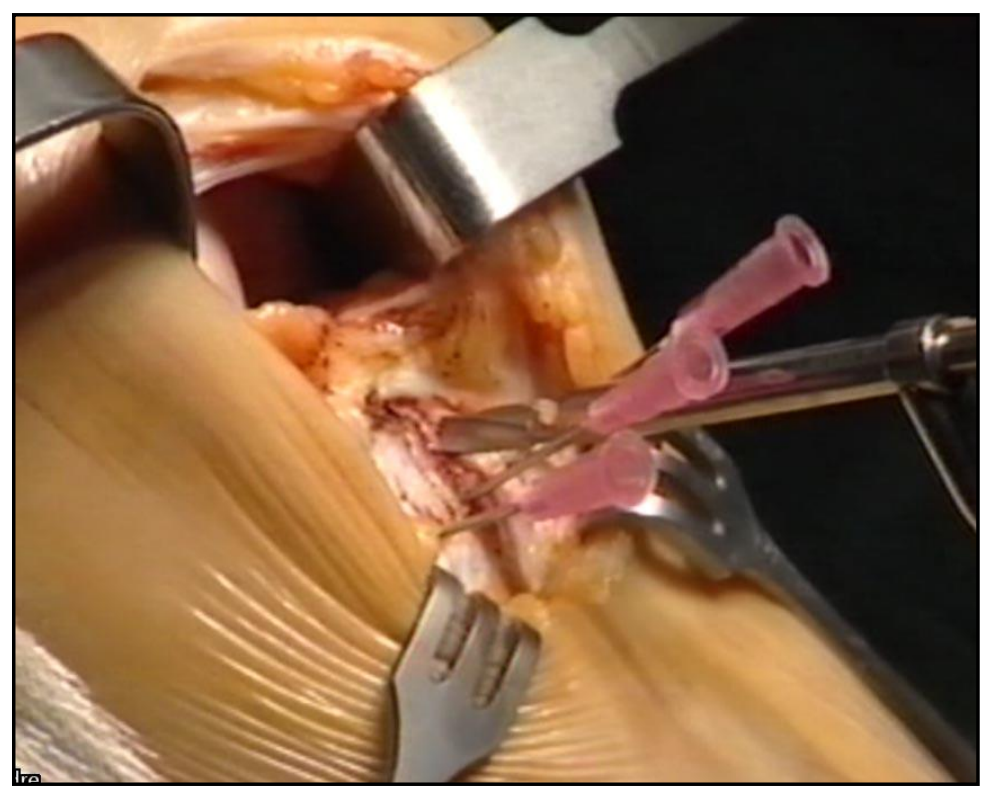

Fig 2:- Repérage du cartilage de croissance à l'aide d'aiguilles fines et forage du tunnel tibial. 


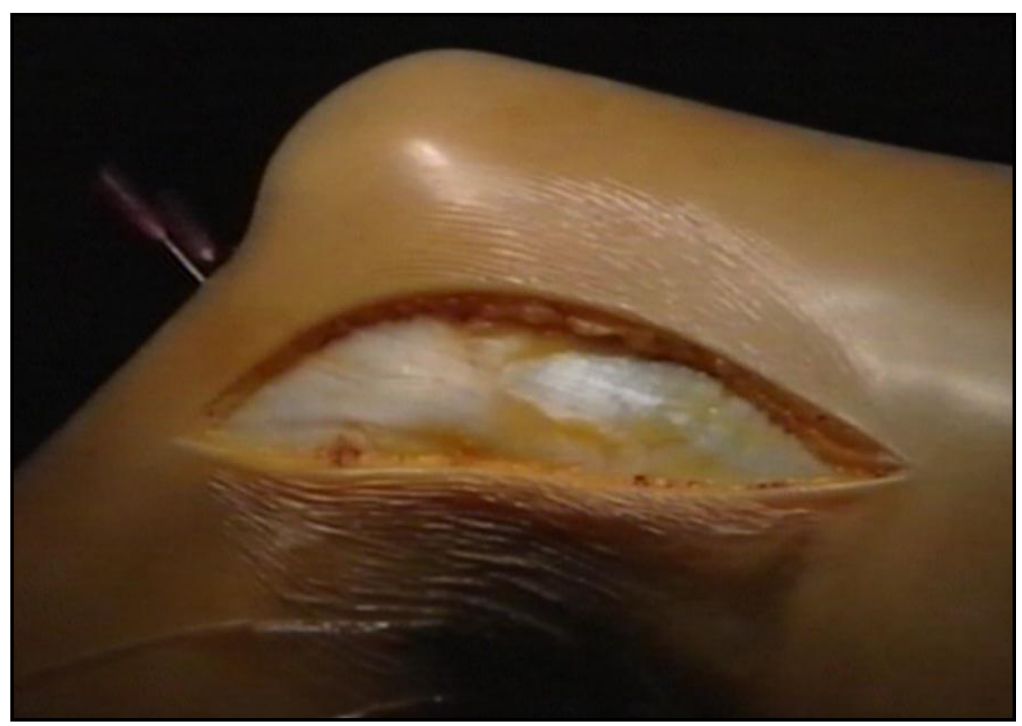

Fig 3:- Incision latérale et exposition du fascia-lata

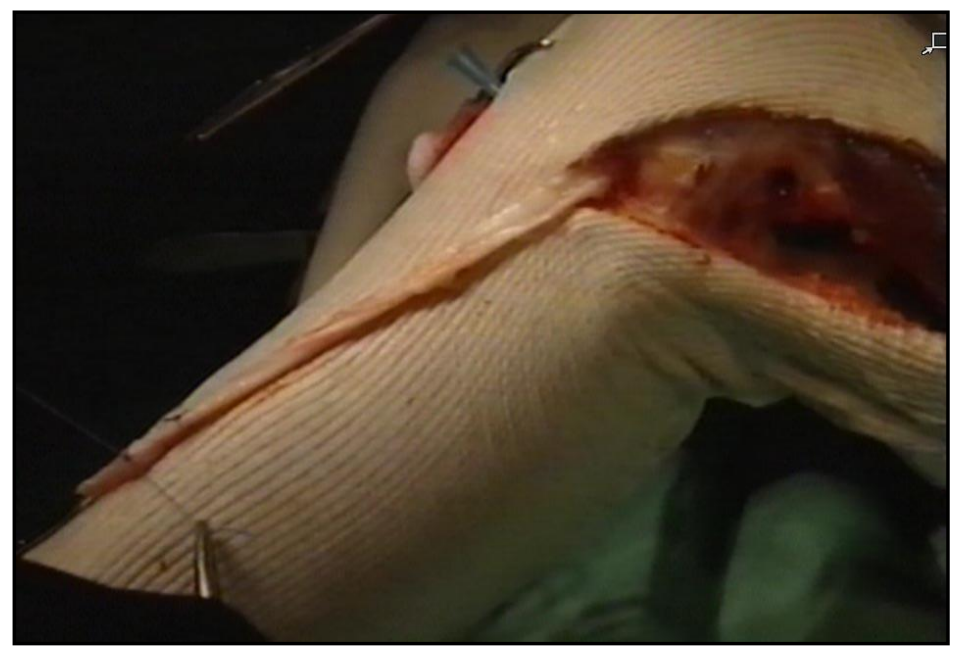

Fig 4:- Prélèvement et tubulisation du transplant avec fil tracteur à son extrémité.

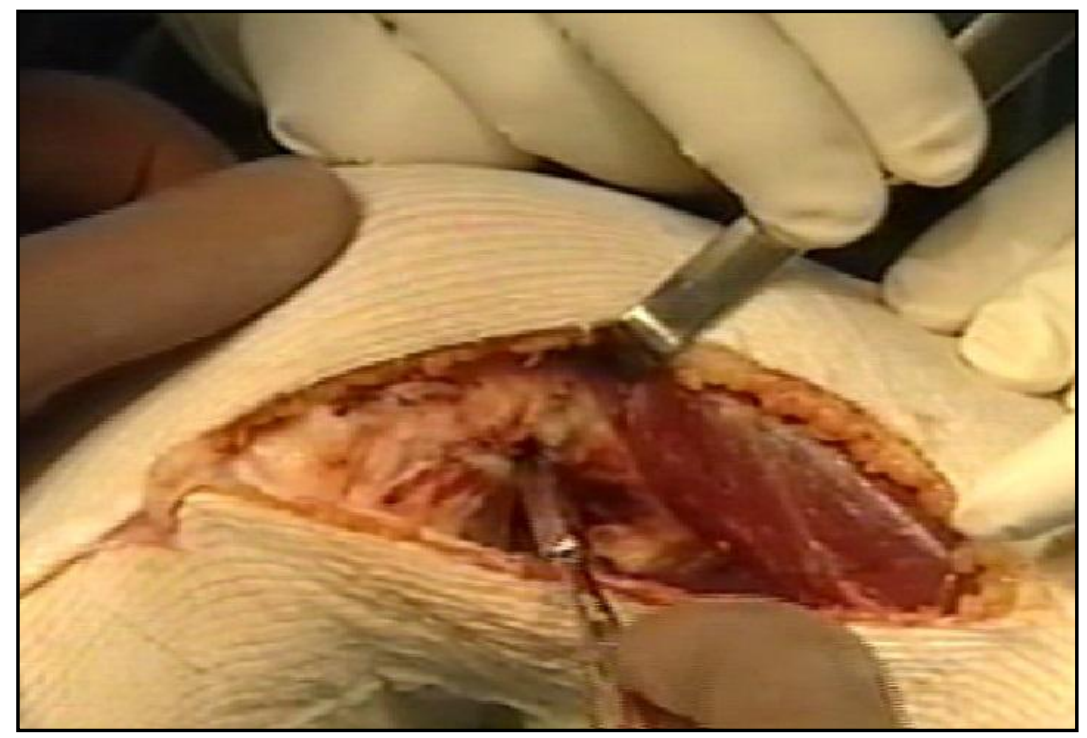

Fig 5:- Réalisation d'une rigole condylienne fémorale en sous périosté. 


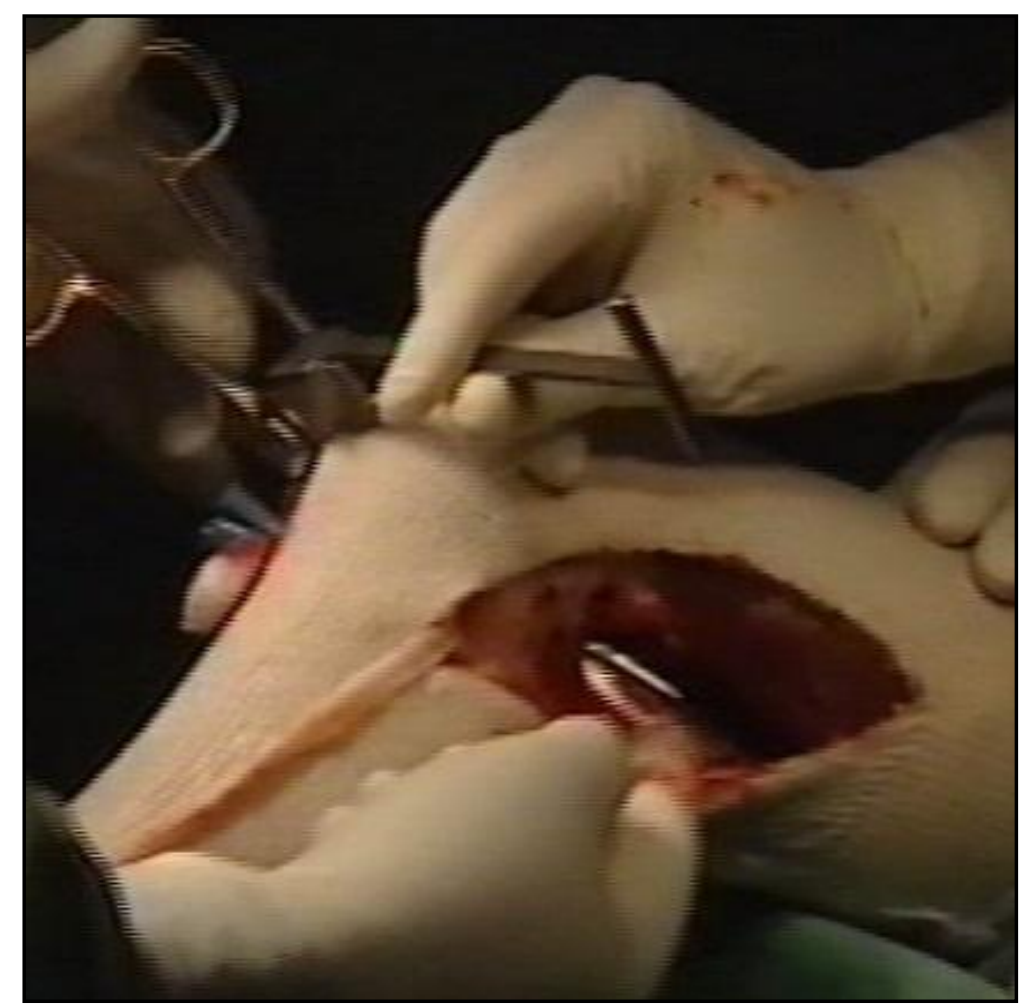

Fig 6:- Perforation de la capsule articulaire postérieure.

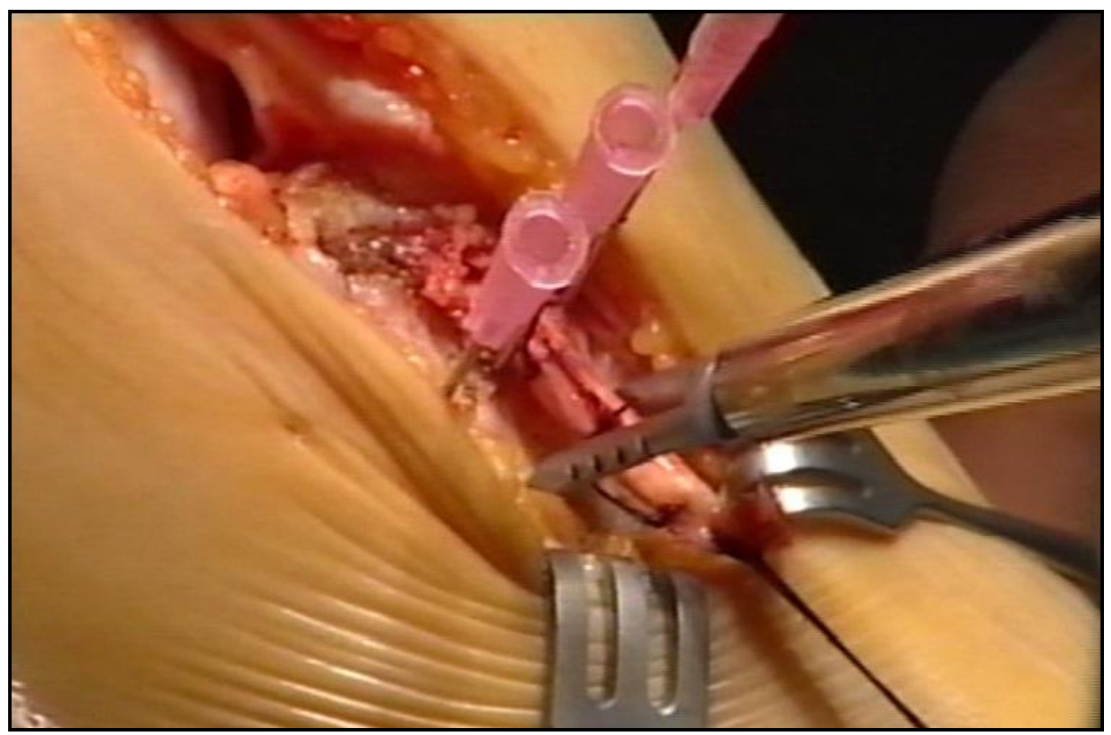

Figure 7 : Passage et fixation du transplant par agrafe métallique positionnée sous le cartilage de croissance.

\section{Discussion:-}

\section{Resultats cliniques:-}

Il n'y a pas dans notre série de douleur antérieure comme c'est le cas dans les plasties au tendon rotulien où ces douleurs sont estimées entre 19\% [2] et 38\% [3] selon les séries et rendent dans 53\% des cas la marche sur les genoux impossible. Les séries de patients analysées dans le service pour le Mac Intosh au fascia-lata par arthrotomie retrouvaient des mobilités à la $6^{\text {ème }}$ semaine de $100^{\circ}$ en moyenne [4]. A 6 semaines un déficit d'extension est retrouvé chez 1 patient, il est de $1,15^{\circ}$ sans qu'il y ait de différence avec le programme de rééducation entre les 
patients. Un arrêt dur, retardé, au test de Trillat-Lachman a été retrouvé chez 5 patients $(21,7 \%)$ à un an d'évolution. Pour Balabaud [5], le Trillat-Lachman est coté à cinq ans à une croix dans $28 \%$ des cas et deux croix pour 10\% des patients. Il n'y a pas de ressaut rotatoire à la manœuvre du "Jerk test" à un an. C'est en accord avec les résultats des plasties mixte dans la littérature. Pour la ligamentoplastie au fascia lata selon Mac Intosh modifiée JH Jaeger", le pourcentage de "Jerk test" négatif est de 100\% à un an, 96\% à cinq ans pour Balabaud [6] et de 98,8\% avec 6,7 ans de recul moyen pour Dodelin. De même pour les ligamentoplasties au tendon rotulien avec retour externe, Stapleton et al. [7] et Jorgenson et al [8] rapportent 96\% d'absence de ressaut rotatoire.

Dans cette dernière série une mesure arthrométrique au KT 2000 a été réalisée et retrouve à $89 \mathrm{~N}$ une différence inférieure à $3 \mathrm{~mm}$ dans $84 \%$ des cas à 6 mois et $86 \%$ à 5 ans. Il y a donc une détente de la greffe dans les premiers mois puis une stabilisation qui se maintient dans le temps. Ce petit jeu articulaire constaté lors de tests statiques a toutefois peu d'influence sur les résultats fonctionnels. Noyes et al [9] rapportent que 12\% de ces patients ont un jeu de plus de $3 \mathrm{~mm}$ par rapport au genou controlatéral alors que plus de la moitié n'a aucune plainte avec un score de Lysholm moyen de 100 points et aucun ressaut. L'évaluation du résultat objectif devrait donc être testée en dynamique plutôt qu'en statique comme le suggère Lefebre et al. [10]. Pour notre étude, la mesure des laxités au KT 2000, chez les 23 patients revus en consultation dont le genou controlatéral était sain, a retrouvé une laxité différentielle moyenne de $2,1 \mathrm{~mm}(0$ à $6 \mathrm{~mm})$ à un an.

\section{Les complications:-}

Un patient a présenté un hématome de cuisse au site du prélèvement, on a relevé un cas de sepsis superficiel. Ces complications ne compromettent pas le pronostic fonctionnel quand elles sont prises en charge à temps. Ainsi ces deux patients ont une mobilité normale et ont pu reprendre leurs activités sportives antérieures au sixième mois post opératoire comme prévu.

\section{La technique chirurgicale:-}

L'affaiblissement du hauban externe, évoqué par certains auteurs, est à nos yeux évité par la réparation systématique du fascia-lata en fin d'intervention [11]. Cette réparation étant devenue toujours possible grâce à la section du septum fémoral latéral proposée par J.H. Jaeger [12]. Le préjudice esthétique a été également mis en avant. Or, actuellement il est possible de réaliser cette technique avec une incision latérale de 6 à $8 \mathrm{~cm}$ associée à une miniarthrotomie. Il n'y a pas d'intervention proposant des cicatrices plus petites qui associe une plastie intra et extraarticulaire.

L'expérience du service en matière de ligamentoplastie nous a conduits à choisir le Mac Intosh au fascia-lata car avec un même transplant nous pouvons réaliser une plastie intra et extra-articulaire équilibrée. En effet le fait que les 2 plasties ne soient pas indépendantes l'une de l'autre évite d'éventuelles différences de tension qui pourraient rendre l'une d'elle inutile.

Le retour externe systématique avec cette technique joue selon nous un rôle fondamental par son effet «anti-jerk » et son rôle protecteur de la plastie intra-articulaire pendant la phase de nécrose des 6 à 12 semaines. Même si Noyes et coll [9], en 2003, n'ont pas montré de différence significative dans les résultats à long terme entre une plastie au tendon rotulien seul et une plastie intra et extra-articulaire, le sujet fait toujours débat puisqu'une conférence de la SOFCOT 2006 y sera consacrée.

Un autre avantage du Mac Intosh au fascia-lata est le diamètre du transplant de $6 \mathrm{~mm}$ qui permet :

1. De conserver l'environnement synovial autour du transplant et ainsi de favoriser sa ligamentisation et de préserver les éventuels mécanorécepteurs restés fonctionnels au sein d'un ligament croisé antérieur cicatrisé en nourrice.

2. D'éviter les conflits avec l'échancrure fémorale qui exposent aux risque de distensions secondaires de la plastie ou de raideurs, voire de ruptures ou pour le tendon rotulien de cyclope syndrome.

3. De réaliser des tunnels osseux de diamètre moins important.

En effet, des études biomécaniques faites par moyen et coll. [11] et par Harner et coll. (12) ont montré que le transplant du droit interne et gracilis a une surface de section de presque 2 fois la surface du ligament croisé antérieur. Ce qui accroît le risque potentiel de conflits avec l'échancrure fémorale.

Nous expliquons également nos bons résultats précoces par le fait que nous sommes partisans d'une rééducation «agressive » [13]. En effet les patients débutent la rééducation au $1^{\mathrm{er}}$ jour post-opératoire, le travail porte sur les 
mouvements actifs et passifs du genou, sur la levée de la sidération du quadriceps permettant le verrouillage actif du genou. 2 à 3 fois par jour les patients sont installés sur des arthromoteurs pour une durée totale d'environ de 2 heures par jour.

\section{Conclusion:-}

L'insuffisance des résultats du traitement conservateur des ruptures du ligament croisé antérieur de l'enfant justifie la nécessité du traitement chirurgical. La procédure chirurgicale réalisée doit respecter le cartilage de croissance. Si la technique est bien conduite, le risque de retentissement sur la croissance est moindre car la majorité des troubles rencontrés dans la littérature est secondaire à des fautes techniques. La technique de Jaeger mérite une place de choix dans la mesure où les résultats sont constamment excellents, lorsque les indications sont bien posées et la technique est conduite de façon rigoureuse.

\section{Conflits d'intérêts:-}

Les auteurs ne déclarent aucun conflit d'intérêts

\section{References:-}

1. Bergerault F, Bonnard C, Courtivron B. Rupture du ligament croisé antérieur chez l'enfant. EMC, techniques chirurgicales: Orthopédie-Traumatologie. Elsevier SAS. 2011; 99: 44-786.

2. Acquitter Y, Hulet C, Locker B, Delbarre JC, Jambou S, Vielpeau C. Intérêt d'une plastie extra-articulaire dans le traitement des laxités antérieures chroniques du genou par une autogreffe de tendon rotulien. Rev Chir Orthop Reparatrice Appar Mot. 2003; 89(23): 413-422.

3. Bak K, Jørgensen U, Ekstrand J, Scavenius M. Results of reconstruction of acute ruptures of the anterior cruciate ligament with an iliotibial band autograft. Knee Surg Sports Traumatol Arthrosc. 1999; 7(1): 111-117.

4. Clancy WG, Nelson DA, Reider B. Anterior cruciate ligament reconstruction using onethird of the patellar ligament, augmented by extra-articular tendon transfers. J Bone Joint Surg (Am). 1982; 64(11): 352-359.

5. Vergis A, Hindriks M, Gillquist J. Saggital plane translation of the knee in anterior cruciate deficient subjects and controls. Med Sci Sports Exer. 1997; 29(5): 1561-1566.

6. Meyer-Fleck D, Chapotot D, Balliet JM, Jaeger JH. Intérêt de l'évaluation isocinétique dans les laxités chroniques du genou traitées chirurgicalement par plastie à l'aide d'un transplant au fascia lata dans Ligamentoplastie du LCA: Mc Intosh FL versus KJ et DIDT. JH Jaeger, C Lutz, W Van Hille. Edition Sauramps medical, 2002; 6(2): $85-91$.

7. Stapleton TR. Complications in anterior cruciate ligament reconstructions with patellar tendon grafts. Sports med an arthroscopy. 1997; 5(1): 156-162.

8. Jørgensen U, Bak K, Ekstrand J, Scavenius M. Reconstruction of the anterior cruciate ligament with the iliotibial band autograft in patients with chronic knee instability. Knee Surg, Sports Traumatol, Arthrosc.2001; 9(5): 137-145.

9. Noyes FR, Butler DL, Grood ES, Zernicke RF, Hefzy MS. Biomechanical analysis of human ligament grafts used in knee ligament repairs and reconstructions. J Bone Joint Surg [Am]. 1984, 66(3): 344-352.

10. Lefebre Y. Etude des prospriètés mécaniques de trois transplants utilisables dans la reconstruction du ligament croisé antérieur. Thèse de médecine, université Louis-Pasteur de strasbourg. 2000; 85(A): 36 - 45.

11. Moyen B. Biomécanique du tendon rotulien et du complexe semi-tendineux, gracilis. Annales de la SFA. 1999; 15(3): $30-33$.

12. Harner CD, Xerogeanes JW, Livesay GA, Carlin GJ, Smith BA, Kusayama T, Kashiwaguchi S, Woo SL. The human posterior cruciate ligament complex: an interdisciplinary study ligament morphology and biomechanical evaluation. Am J Sp Med. 1995; 6(2): 736 - 745.

13. Seynaeve P, Klupczynski D, Ruiz C. Réeducation après chirurgie ligamentaire du genou : protocole Mac Intosh modifié au fascia lata par JH Jaeger. Livre : Ligamentoplastie du LCA : Mac Intosh FL versus KJ et DIDT, réparation cartilagineuse. Sauramps médical, 2002; 6(2): 56 - 60. 